\title{
Auditory temporal processing deficit in dyslexia is associated with enhanced sensitivity in the visual modality
}

\author{
Sabine Heim, ${ }^{\text {CA }}$ Robert B. Freeman Jr, Carsten Eulitz and Thomas Elbert \\ Department of Psychology, University of Konstanz, P.O. Box D25, 78457 Konstanz, Germany \\ ${ }^{\mathrm{CA} C o r r e s p o n d i n g ~ A u t h o r ~}$
}

\begin{abstract}
Developmental dyslexia has been associated with a deficit in temporal processing, but it is controversial whether the postulated deficit is pansensory or limited to the auditory modality. We present psychophysical assessment data of auditory and visual temporal processing abilities in children with dyslexia. While none of the dyslexic children displayed temporal processing abnormalities in the visual sensory mod-
\end{abstract}

ality, dyslexics with poor auditory temporal scores reached high-level visual performance. Our results do not confirm the hypothesis of a general temporal processing deficit for dyslexia but suggest that limitations in auditory temporal processing might be compensated for by a well-functioning visual sensory modality.

Key words: Auditory; Children; Dyslexia; Temporal processing; Visual

\section{INTRODUCTION}

Developmental dyslexia is characterized by a failure in learning to read, spell and write despite normal intellectual capacity, educational resources, and adequate sociocultural opportunity, even though sensory deficits, neurological pathology, and other impediments to attaining literacy skills have been ruled out [1,2]. Psychoacoustic studies have shown that many children with language and literacy problems display limitations in reception of brief and rapidly changing phonemes (e.g. stop consonant syllables) or rapidly successive sound inputs [3,4] for which Paula Tallal has been using the term temporal processing defi-

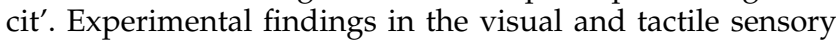
modalities led some researchers to conclude that a temporal processing deficit might be pansensory in children with language impairment and people with dyslexia [3,4]. Because multimodal approaches within a single study have been sparse and inconclusive, the question of whether a temporal processing deficit in these populations might be general or modality specific has been a subject of intense debate.

The present study aimed to investigate temporal processing abilities in both the auditory and visual modality in children with dyslexia and normally literate controls. The auditory task required a same-different judgment of two successively presented stop consonant-vowel (CV) syllables (/ba/ and /da/), which are characterized by rapid frequency changes (formant transitions) that occur during the initial few tens of milliseconds. As a control condition, the same syllables were presented with temporally ex- tended formant transitions [5]. This control procedure should provide an evaluation of the auditory temporal processing hypothesis for dyslexia: a relative weakness in discriminating between $\mathrm{CV}$ syllables with rapidly changing formant transitions compared with $\mathrm{CV}$ syllables with temporally extended formant transitions would support the view of a deficit in temporal acoustic processing; poor performance on both conditions would suggest that the difficulties experienced by the dyslexic children are speech-specific in general. In accordance with Tallal's work, performance on the two conditions was measured at different interstimulus intervals (ISIs) varying between 8 and $305 \mathrm{~ms}[5,6]$. In the visual task, thresholds for the detection of temporal order of two light flashes were determined. The findings of the study should add valuable information to the issue of whether a temporal processing deficit in dyslexia is pansensory or modality specific.

\section{MATERIALS AND METHODS}

We tested 22 children (three females) diagnosed with developmental dyslexia and 11 controls (one female) with normal literacy skills who matched the others in mother tongue (all native speakers of German), age and non-verbal intelligence (Table 1). Sixteen children from the dyslexic group attended a special school for dyslexia, the other six having been referred by an independent institute that specializes in diagnosing and treating children with learning disabilities. Although the dyslexic subjects were receiving remedial training and intense tutoring, they performed significantly worse on measures of reading, phonological 
Table I. Psychometric data for study groups: means \pm s.d.

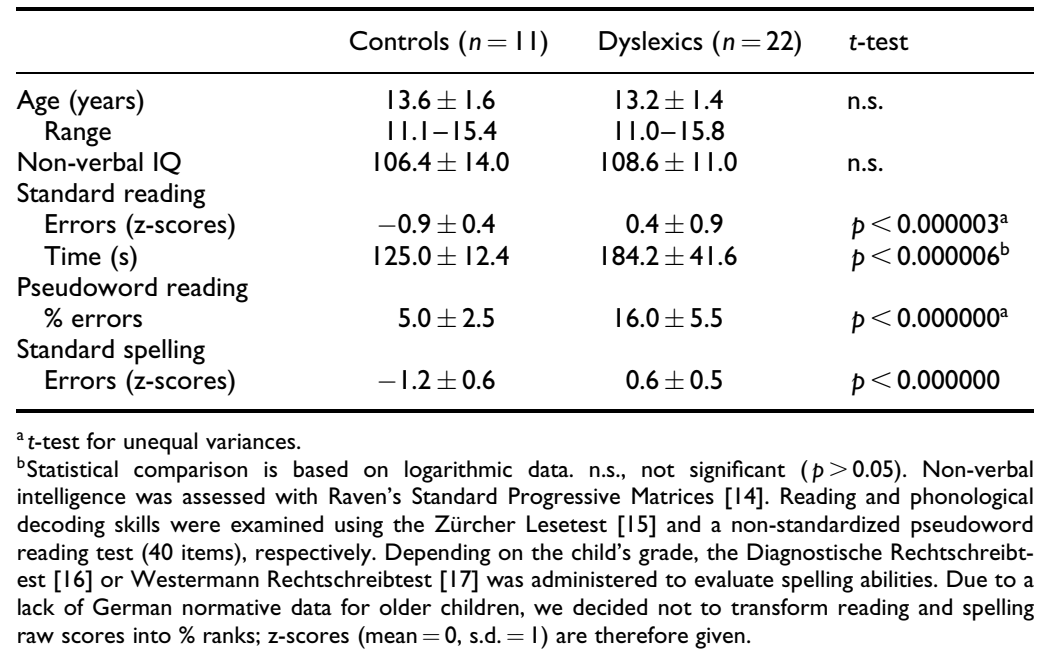

decoding and spelling than the controls (Table 1). All subjects had normal hearing thresholds and normal or corrected-to-normal visual acuity. Informed consent was obtained from the parents of the children. All children were rewarded with shopping vouchers or cinema tickets for their voluntary participation.

The psychophysical tasks were carried out on an IBM compatible 486 PC. The order of the tasks was counterbalanced across subjects. Each child was tested individually in a quiet room.

Auditory same-different task: The German CV syllables /ba/ and /da/ were created with a sampling rate of $10 \mathrm{kHz}$ in a cascade mode by using Speechlab software [7] based on a Klatt cascade/parallel formant synthesizer [8]. The total stimulus duration was $250 \mathrm{~ms}$ including a formant transition (FT) period of either $40 \mathrm{~ms}$ (rapid FT condition) or $90 \mathrm{~ms}$ (extended FT condition). The fundamental frequency of each syllable started at $128 \mathrm{~Hz}$ and decreased linearly to $109 \mathrm{~Hz}$ at stimulus offset. The syllables were composed of three formants (F1-F3) and differed in the onset frequencies of the second and third FT. The starting points of the second and third FT were $1095 \mathrm{~Hz}$ and $2100 \mathrm{~Hz}$ for the syllable / ba/ and $1702 \mathrm{~Hz}$ and $2633 \mathrm{~Hz}$ for $/ \mathrm{da} /$. The steady-state formant frequencies of the vowel /a/ were 770, 1340, and $2400 \mathrm{~Hz}$ for F1, F2, and F3, respectively. The amplitude of voicing was constant at $54 \mathrm{~dB}$ and fell linearly to $11 \mathrm{~dB}$ during the last $25 \mathrm{~ms}$ of the stimulus.

The experimental procedure was identical for the rapid and extended FT conditions. The syllables were delivered to both ears via Sony MDR-CD470 headphones at $\sim 72 \mathrm{~dB}$ sound pressure level. The task required the child to press the right (green) panel if two successive syllables (/ba/$/ \mathrm{ba} /, / \mathrm{da} /-/ \mathrm{da} /$ ) were the same and the left (red) panel if they were different $(/ \mathrm{ba} /-/ \mathrm{da} /, / \mathrm{da} /-\mathrm{ba} /)$ by using the index finger of her/his right hand. Feedback was provided after each stimulus pair (trial) by a happy or unhappy face on the computer screen. The intertrial interval was $2 \mathrm{~s}$. The training phase consisted of a maximum of
72 trials (18 for each possible pair combination, randomly intermixed) and finished earliest if a criterion of 30 correct responses in 36 consecutive trials was achieved. The task was terminated for subjects who did not meet the criterion. During training an ISI (defined as syllable offset to onset) of $428 \mathrm{~ms}$ was employed; in the immediately following testing phase syllables were presented at six different ISIs: $8,15,30,60,150$ and $305 \mathrm{~ms}[5,6]$. Testing included 48 trials, eight $(2 \times 4$ syllable pair combinations) for each ISI, with a randomized presentation of the different intervals. The percentages of correct trials were measured at each ISI.

Visual temporal order task: Equiluminant light flashes of green and red were generated by two light-emitting diodes (LEDs). LEDs were mounted side by side (distance $1 \mathrm{~cm}$ ) on a black surface slanted toward the subject. The LED apparatus was positioned on a table $(\sim 70 \mathrm{~cm}$ high) with a constant viewing distance of $40 \mathrm{~cm}$. Subjects were asked to press either a red (left) or green (right) key on the computer keyboard with the index finger of their right hand indicating the LED which flashed first. After the second LED flashed, both LEDs remained on for $2 \mathrm{~s}$. Twoelement stimulus sequences (red-green, green-red) were randomized across trials. The intertrial interval was $5 \mathrm{~s}$. In order to direct subject's attention to the LEDs, each trial was announced by a brief tone. During an initial training phase of 10 trials, onsets between two light flashes (SOA, stimulus onset asynchrony) varied between 310 and $400 \mathrm{~ms}$, i.e. rather long intervals, to allow all subjects to understand the task. In the testing phase (40 trials) the SOA was adjusted from trial to trial (starting SOA = $300 \mathrm{~ms}$ ), using an adaptation of the staircase procedure [9]. After one correct response in a given staircase, the SOA was shortened, whereas an incorrect answer led to the SOA being lengthened. Sizes of downward or upward steps (reversals) were $10 \%$ of the previous value; below a $10 \mathrm{~ms}$ SOA a step size of $1 \mathrm{~ms}$ was used. The visual temporal-order threshold in milliseconds, defined as the arithmetic mean of the last 20 reversals, was calculated automatically by the computer program. 


\section{RESULTS}

Univariate ANOVA showed no significant group effect on visual temporal-order thresholds, indicating that both the dyslexic (mean ( \pm s.e.m.) threshold $8.9 \pm 1.2 \mathrm{~ms}$ ) and control children (threshold $9.9 \pm 1.3 \mathrm{~ms}$ ) managed the visual temporal processing task without difficulty and equally well.

Mixed-design ANOVA performed on the data of auditory processing revealed a significant group main effect $(\mathrm{F}(1,31)=4.2, p<0.050)$ for the dyslexics to have lower scores than the controls in both FT conditions across the range of ISIs tested (Fig. 1). Even though no significant interaction was obtained with the factor group there was a small tendency for larger group differences in the rapid than in the temporally extended FT condition. As demonstrated by other investigators, some people with dyslexia may have no difficulties in tasks involving rapidly changing acoustic stimuli [3,4]. This might also apply to a subset of dyslexic children in the present study and in turn have weakened the group $\times$ condition interaction. Therefore, the dyslexic subjects were subclassified into two groups based on their discrimination performance on the rapidly changing syllables in the three shortest ISIs $(8,15$ and $30 \mathrm{~ms}$ ). Dyslexic subjects scoring $\geqslant 87.5 \%$ in two of the shortest ISIs were classified as good perceivers, otherwise they were classified as poor perceivers. Mixed-design ANOVA carried out with these groups yielded a significant group $\times$ FT condition interaction effect $(\mathrm{F}(2,30)=6.8, p<0.004)$.

As illustrated in Fig. 2, the 14 dyslexic subjects comprising the group of good perceivers did not differ from the controls in any of the conditions. However, the poor-

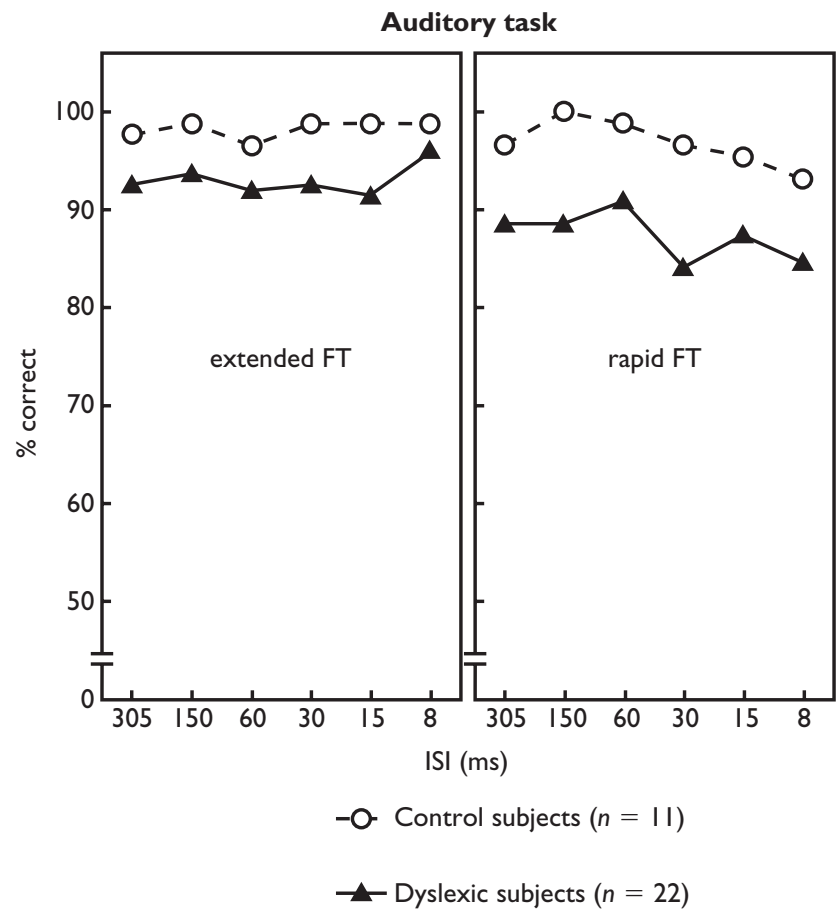

Fig. I. Percentage correct for II control (open circles) and 22 dyslexic (filled triangles) subjects on the auditory same-different task at various ISIs of the rapid and extended FT conditions.

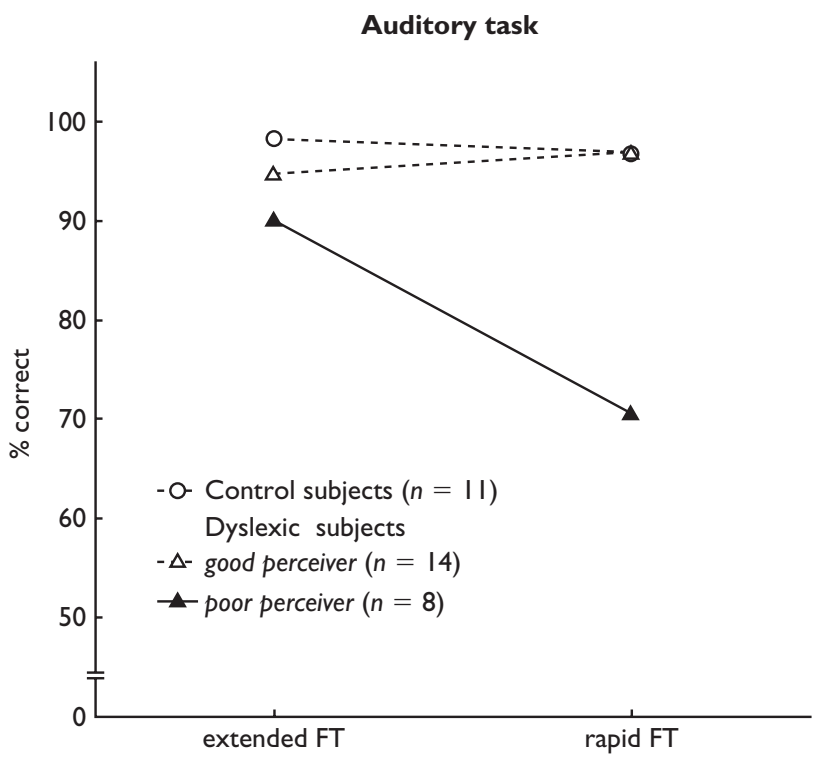

Fig. 2. Percentage correct on the auditory same-different task in the control subjects (open circles) and in dyslexic subjects who were subclassified as good (open triangles) and poor (filled triangles) perceivers according their performance in the three shortest ISIs of the rapid FT condition.

perceiver group ( $n=8$ dyslexic subjects) was significantly less accurate in the rapid FT condition compared to the other groups (Scheffé's $p<0.001$ ) and their performance level in the extended FT condition (Scheffé's $p<0.018$ ). This result suggests that at least 8 subjects of the dyslexic group displayed difficulties in processing rapid temporal acoustic information.

In order to avoid possible ceiling effects, we used the minimum correct percentages of each subject attained at any ISI of the rapid FT condition for analyzing the relationship between auditory and visual temporal processing. Individual subject data for minimum auditory scores in relation to visual temporal-order thresholds are presented in Fig. 3.

A bivariate prediction analysis based on weighted Kappa indicated a significant distribution pattern for the dyslexic group $(p<0.004)$. Ten dyslexic subjects whose performance in the same-different task pointed to limitations in auditory temporal processing (i.e. $\leqslant 75 \%$ correct [10]), demonstrated low temporal-order thresholds $(<10 \mathrm{~ms})$ in the visual sensory modality. In five of these children, visual thresholds were $>1$ s.d. below the group mean of normal controls. In control subjects, the statistical relationship between measures on auditory and visual temporal processing did not reach significance.

\section{DISCUSSION AND CONCLUSION}

Our results reveal an auditory temporal processing deficit in children with dyslexia, although temporal sensitivity was enhanced rather than impaired in the visual task. Thus, the current data provide no evidence for a pansensory or general temporal processing deficit in children with 


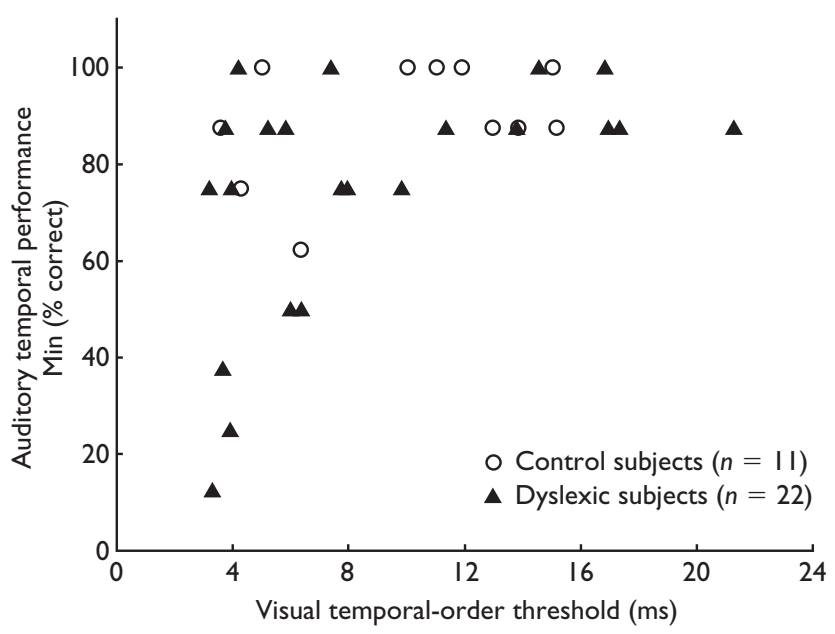

Fig. 3. Plot of auditory temporal performance in relation to visual temporal-order thresholds (ms) for II control (open circles) and 22 dyslexic (filled triangles) children. Min (\% correct) denotes minimum correct percentages attained at any ISI of the rapid FT condition. Low visual thresholds reflect superior performance.

dyslexia. On the contrary, poor auditory temporal sensitivity might be compensated by a well functioning visual sensory modality. Supportive evidence has been provided by a study by Talcott et al. [11] in a sample of unselected elementary school children: auditory and visual temporal processing were found to be differently engaged in phonological and orthographic skills, implying independent candidates in determining a child's ability to learn to read. Our findings support the view that intervention methods for dyslexia should target the auditory modality [12,13].

\section{REFERENCES}

1. Miles T. Dyslexia: The Pattern of Difficulties. Springfield, IL: Charles C. Thomas, 1983.

2. Dilling $\mathrm{H}$, Mombour $\mathrm{W}$ and Schmidt MH. International Classification of Mental Diseases. ICD-10. Bern: Huber, 1991.

3. Tallal P, Miller S and Fitch RH. Ann NY Acad Sci 682, 27-47 (1993).

4. Farmer ME and Klein RM. Psychon Bull Rev 2, 460-493 (1995).

5. Tallal P and Piercy M. Neuropsychologia 13, 69-74 (1975).

6. Tallal P and Piercy M. Neuropsychologia 12, 83-93 (1974).

7. Diesch E. Behav Res Methods Instr Comput 29, 302 (1997).

8. Klatt DH. J Acoust Soc Am 67, 971-995 (1980).

9. Cornsweet TN. Am J Psychol 75, 485-491 (1962).

10. Tallal P and Piercy M. Nature 241, 468-469 (1973).

11. Talcott JB et al. Proc Natl Acad Sci USA, 97, 2952-2957 (2000).

12. Merzenich MM et al. Science 271, 77-81 (1996).

13. Tallal P et al. Science 271, 81-84 (1996).

14. Heller KA, Kratzmeier $\mathrm{H}$ and Lengfelder A. Standard Progressive Matrices von J. C. Raven. Göttingen: Beltz, 1998.

15. Linder M and Grissemann H. Zürcher Lesetest, ZLT. Bern: Huber, 1998.

16. Grund M, Haug G and Naumann CL. Diagnostischer Rechtschreibtest für 5. Klassen, DRT 5. Weinheim: Beltz, 1995.

17. Rathenow P, Laupenmühlen D and Vöge J. Westermann Rechtschreibtest 6+, WRT 6+. Braunschweig: Westermann, 1981.

\footnotetext{
Acknowledgements: We thank $\mathrm{H}$. Vlahu for help in data collection, W. Nagl for advice on statistical analysis, P. Dixon for important contributions towards programming the LEDs, and A. Keil for comments on the manuscript. This work was supported by grants from the Deutsche Forschungsgemeinschaft.
} 